\title{
Métropolisation, migrations internationales et pluralité des espaces sociaux: les agglomérations suisses face au défi de l'intégration
}

\section{Antonio Da Cunha, Lausanne}

\section{Introduction}

La région métropolitaine est la figure contemporaine du processus d'urbanisation. Les villes changent de morphologie et d'échelle. Certains ont avancé l'idée d'une urbanisation généralisée sans forme, sans limites et sans distinction (AsCHER 1995; CORBOZ 2000). L'observation tend à montrer que le nouveau régime de métropolisation a plutôt tendance à produire des reconfigurations de l'espace urbain marquées par des polarisations spatiales et sociales et par une amplification des phénomènes de ségrégation résidentielle (MARTENS \& VERVAEKE 1997). Les grandes agglomérations apparaissent divisées démographiquement, socialement et culturellement et les modalités actuelles du développement urbain semblent accentuer ces clivages.

Le monde est devenu plus petit et les territoires sont de plus en plus interdépendants. L'ère de la globalisation est aussi celle des migrations internationales. Au cours des dernières décennies, les populations européennes se sont profondément renouvelées grâce aux flux migratoires. Les opportunités d'interaction spatiale permises par la contraction de l'espacetemps métamorphosent les systèmes de peuplement. Un nouveau régime d'urbanisation se met en place combinant des processus se déployant à différentes échelles spatiales. A l'échelle du réseau urbain, le régime métropolitain se caractérise par la consolidation du rôle directionnel des grandes agglomérations. A l'échelle locale, il se traduit par la formation d'espaces urbanisés de plus en plus étalés, hétérogènes et fragmentés du point de vue physique, social et politique.

Cet article a pour objectif la mise en évidence de certains aspects du processus d'installation des migrants et des modalités de leur inclusion dans l'espace résidentiel des cinq grandes agglomérations suisses (Photo 1). Si, pour certains observateurs (PIgUet 2004), l'intégration des populations étrangères peut être considérée comme une "sucess story», l'ethnicisation des villes pose de nouveaux défis à la gestion des différences socioculturelles dans un contexte de précarisation sociale et de durcissement politique. Nous sommes convaincus que la réponse à la double question de la fragmentation socio-culturelle et de la montée de la xénophobie passe par une conception de l'intégra- tion associant Etat-social et démocratie intégrative à l'échelle locale. Dans le champ politique, la question sociale et la question de la cohabitation de personnes ayant des origines multiples ne peuvent plus être dissociées. Dans cette perspective, les agglomérations urbaines sont appelées à mettre en œuvre des actions transversales visant à gérer la diversité, à renforcer le lien social et à élargir l'exercice de la citoyenneté.

\section{Métropolisation et migrations internationales: ségrégation urbaine et précarité sociale}

En Europe, la globalisation, les disparités de développement, les crises économiques, les guerres et la violence ont alimenté des flux croissants de migrants d'origines diverses. Les migrants représentent une partie non négligeable de la croissance démographique annuelle. Comme partout en Europe, l'intensification des migrations internationales s'accompagne en Suisse d'une diversification de la figure de l'immigré. L'articulation de ces dynamiques démographiques globales au fonctionnement des marchés immobiliers des grandes agglomérations et aux logiques sociales d'autoagrégation résidentielle tend à amplifier les divisions sociales et culturelles de l'espace urbain.

\subsection{Les centres des grandes agglomérations: la porte d'entrée des migrations internationales}

La globalisation des marchés du travail et le pluralisme culturel vont de pair. Selon Haug (1995), le pluralisme croissant de la population résidente étrangère se manifeste de manière particulièrement évidente dans le domaine des langues et des religions. WANNER (2004) met en évidence qu'en 1960, près de $90 \%$ des étrangers résidant en Suisse étaient originaire d'un pays limitrophe, cette proportion ayant diminué à $35 \%$ en 2000. La multiplication des origines nationales, des qualifications et des statuts révèle des transformations du marché du travail autant que la mobilisation des réseaux sociaux dans l'espace élargi des circulations. Une partie non négligeable des migrants s'est sédentarisée ( $75 \%$ des étrangers bénéficient d'un permis d'établissement). Certains se sont naturalisés. Un tiers de la population helvétique est directement issue de la migration (PIGUet 2004). Considérée d'abord comme phénomène économique provisoire, la présence des populations migrantes est devenue un élément constitutif de la société. La diversité des populations fonde indiscutablement la société suisse du XXI' siècle. 


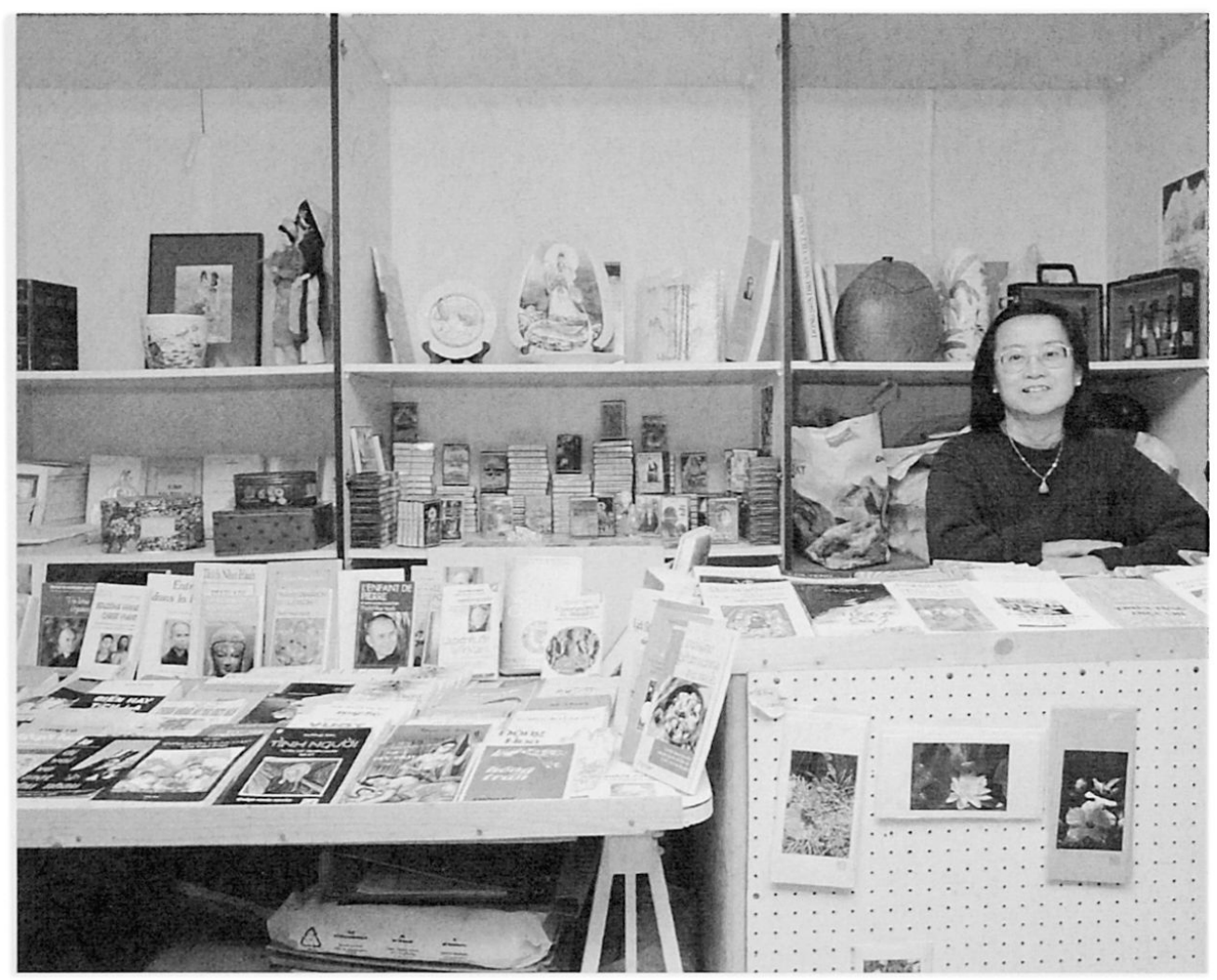

Photo 1: La migration comme exil, parcours ou projet? Un membre de la communauté vietnamienne en Suisse Migration as a form of exile, career step or project? A member of the Vietnamese community in Switzerland Die Migration als Exil, Karriere oder Projekt? Ein Mitglied der vietnamesischen Gemeinde in der Schweiz Photo: L. NGUYEN

Des études récentes (Huissoud et al. 1999) ont mis en évidence, de manière très détaillée, les structures et tendances de la différenciation dans les espaces urbains en Suisse. Le calcul des indices de ségrégation montre notamment que la variable la plus importante en termes de différenciation spatiale entre groupes est celle de l'appartenance culturelle. Les catégories socio-professionnelles et les classes d'âges mettent en évidence des écarts moins significatifs entre les zones centrales, les communes suburbaines et les communes périurbaines. L'analyse du recensement 2000 confirme et précise ce constat général.

L'exploitation des données issues du dernier recensement montre, tout d'abord, que la croissance des grandes agglomérations (Zurich, Bâle, Genève, Berne, Lausanne) est fortement alimentée par des flux migratoires en provenance d'autres pays (DA CUNHA \& BOTH 2004). La population totale des cinq grandes agglomérations en 2000 est de 2'691'887 personnes. Entre 1970 et 2000, les noyaux ont perdu 147'560 habitants, passant ainsi de $46.9 \%$ à $35.7 \%$ de la population totale des cinq agglomérations. Cependant, ces villes centrales constituent toujours un lieu privilégié d'établissement des migrants. La population étrangère représente $25.4 \%$ de la population résidente totale des cinq grandes agglomérations. La répartition intra-agglomération des populations suisses et étrangères s'inscrit selon un gradient centre-périphérie: les étrangers résidant dans les communes-centres des agglomérations constituent $44.9 \%$ du total de la population étrangère installée dans les cinq agglomérations. Cette proportion est beaucoup plus modeste dans les communes périurbaines (11.6\%) alors que celles-ci représentent $19.4 \%$ de la population des cinq agglomérations. Les migrations internationales jouent un rôle majeur dans la dynamique démographique des grandes agglomérations et tout particulièrement de leurs centres: les cinq villes-centres des grandes agglomérations représentent $13.2 \%$ de la population résidante totale du pays, mais elles captent à elles seules $24.3 \%$ du flux total de la population arrivant de l'étranger (personnes domiciliées à l'étranger en 1995).

A leur arrivée en Suisse, les travailleurs étrangers et leurs familles s'installent de préférence dans les grandes villes, au sein des régions métropolitaines, où les opportunités d'emploi semblent être plus importantes, 
notamment dans le secteur des services non qualifiés. Compte tenu de leur situation économique généralement précaire, ils cherchent un logement dans les secteurs les moins onéreux du marché immobilier dans les noyaux des grandes agglomérations. Les zones centrales offrent d'autres avantages: le migrant se sent moins étranger dans une grande ville, au milieu d'autres étrangers, que dans un village socialement homogène. Les nouveaux migrants peuvent y trouver aussi des équipements spécifiques (p.ex.commerces, restaurants, associations ethniques) et des réseaux sociaux généralement plus ouverts. En revanche, la population suisse est plus fortement sur-représentée dans les communes périurbaines qui semblent offrir un cadre de vie plus confortable et plus apaisant, correspondant aux aspirations des ménages plus favorisés et tout particulièrement aux ménages avec des enfants en bas âge ou en âge scolaire (DA CUNHA et al. 2004). Les familles disposant de revenus élevés acquièrent de meilleurs logements et de meilleures conditions d'habitat que celles qui ont des revenus plus modestes. Ce phénomène d'accès sélectif à la propriété et à la location des surfaces résidentielles engage des processus de différenciation qui touchent toutes les catégories socio-démographiques de la population suisse et étrangère.

\subsection{Un modèle de ségrégation multiforme: sélectivité des flux et profils socio-démographiques}

L'exploitation des informations disponibles met en évidence un modèle de ségrégation multiforme: démographique, sociale et ethnique. Sur les 194'686 personnes nouvellement domiciliées dans les villes-centres des agglomérations en 2000,70 '046, soit $36 \%$, arrivent d'un pays étranger et $64 \%$ d'une autre commune suisse. Les migrations internes vers ces centres se répartissent de la manière suivante: $46.0 \%$ arrivent d'une autre commune de la même agglomération, tandis que $54 \%$ proviennent d'une autre zone urbaine ou d'une commune rurale. Ce couplage entre des flux de mobilité internationaux et des flux résidentiels de courte distance ( $44.4 \mathrm{~km}$ en moyenne, en dépit du fait que $37.5 \%$ des migrations internes sont en provenance d'une autre zone urbaine) constitue une caractéristique majeure du modèle de croissance actuel des régions métropolitaines. On retiendra de l'analyse que le profil social des populations actives provenant de l'étranger est double. En effet, les groupes situés aux deux extrémités de l'échelle des stratifications sociales apparaissent fortement sur-représentés (dirigeants et professions intellectuelles: $+42 \%$; travailleurs non qualifiés: $+44 \%)$. Tout autre est le profil des migrations internes (nouveaux arrivés en provenance des autres communes du pays): les employés, les indépendants et les ouvriers sont plus largement représentés.

Le recensement de population ne permet pas de quantifier les sorties vers l'étranger. On se contentera d'ob- server que $64.4 \%$ des «flux sortants» internes se dirigent vers une commune de la même agglomération. Les villes-centres des grandes agglomérations constituent indiscutablement le moteur des dynamiques d'étalement et de différenciation de l'espace social des grandes agglomérations. Tout se passe comme si elles captaient des flux de longue distance pour les déverser ensuite dans leurs enveloppes suburbaines et périurbaines. Cependant, ces mouvements de redistribution démographique sont sélectifs. Les profils des personnes entrant et sortant du noyau des grandes agglomérations sont tout à fait distincts ( $D_{A}$ CUNHA \& Вотн 2004). La caractéristique la plus évidente du modèle de croissance des agglomérations est l'apport démographique des populations étrangères, des jeunes adultes et des personnes seules, à la croissance des villes-centres, d'une part, le déversement résidentiel des couples avec enfants et des populations plus aisées dans les communes suburbaines et périurbaines, d'autre part. Le tableau 1 illustre le tri sélectif opéré à la sortie des noyaux des agglomérations sur les populations déménageant vers les couronnes .

D'un côté de l'échelle sociale, les dirigeants, les professions libérales, les indépendants, les cadres et les intellectuels partent du centre pour s'installer dans les communes périurbaines; de l'autre côté, les travailleurs non-qualifiés, les ouvriers et les employés partent vers les zones suburbaines. Une caractéristique commune semble les réunir: quelle que soit leur position sociale, ils ont généralement des enfants en bas âge ou en âge scolaire.

Les parcours résidentiels se dessinent en fonction des changements de statuts, du cycle de vie des ménages, des comportements et des attitudes face à l'environnement socio-résidentiel. Au risque d'une certaine schématisation, nous pouvons affirmer que les centres des agglomérations tendent à capter des populations d'origine étrangère et des jeunes adultes en formation, alors qu'ils refoulent sélectivement vers les couronnes des «actifs mûrs» et des couples avec enfants. D'un côté, les dirigeants, les professions libérales, les indépendants, les cadres et les intellectuels, plus souvent d'origine suisse et avec des enfants, partent du centre pour s'installer dans les «enclaves périurbaines» peu denses et à habitat individuel prépondérant. De l'autre côté de l'échelle sociale, les travailleurs non qualifiés, les ouvriers et les employés, plus souvent d'origine étrangère et aussi avec des enfants, partent vers les zones suburbaines, plus denses et à habitat collectif dominant. Corrélativement à ce double processus, on observe un émiettement du tissu social des centres des agglomérations où le nombre de ménages isolés d'origine suisse et étrangère (p.ex. célibataires, divorcés, veufs) ne cesse d'augmenter. Les centres et les périphéries suburbaines et périurbaines présentent ainsi 


\begin{tabular}{|l|c|c|c|c|}
\hline Catégories socio-professionnelles & En $\%$ & $\begin{array}{c}\text { Communes } \\
\text { suburbaines } \\
\mathbf{1}^{\text {ere }} \text { couronne }^{*)}\end{array}$ & $\begin{array}{c}\text { Communes } \\
\text { suburbaines } \\
\mathbf{2}^{\text {eme }} \text { couronne }^{*)}\end{array}$ & $\begin{array}{c}\text { Communes } \\
\text { périurbaines }\end{array}$ \\
\hline Dirigeants & 3.4 & 0.94 & 0.86 & 1.25 \\
\hline Professions libérales & 2.6 & 0.66 & 0.56 & 1.42 \\
\hline Autres indépendants & 8.1 & 0.96 & 0.91 & 1.16 \\
\hline Professions intellectuelles et d'encadrement & 20.2 & 0.68 & 0.71 & 1.09 \\
\hline Professions intermédiaires & 24.9 & 0.98 & 0.98 & 1.01 \\
\hline Non-manuels qualifiés: employés & 25.6 & 1.10 & 1.15 & 0.95 \\
\hline Manuels qualifiés: ouvriers & 6.0 & 1.28 & 1.32 & 0.75 \\
\hline Travailleurs non qualifiés & 9.2 & 1.44 & 1.33 & 0.70 \\
\hline
\end{tabular}

*) Indice de localisation/localization index/Lokalisationsindex

Tab. 1: Déménagement d'habitants des villes-centres des grandes agglomérations suisses, à l'intérieur de la même agglomération, selon les catégories socio-professionnelles et les types de communes (1995-2000)

Intra-agglomeration moves of persons in large Swiss agglomerations according to socio-professional categories and urban zones (1995-2000)

Umzïge von Personen aus Zentren der grossen Schweizer Agglomerationen innerhalb derselben Agglomeration, nach sozio-professionellen Kategorien und Gemeindetypen (1995-2000)

des régimes démographiques très différenciés: la mobilité résidentielle, le processus de filtrage et d'écrémage, selon l'origine, le statut socioprofessionnel, la taille et le type des ménages, l'état civil et l'âge, engendrent des géotypes spécifiques.

En bref, les dynamiques globales (migrations internationales) s'articulent à l'échelle des régions métropolitaines avec des processus de filtrage passifs et actifs fondés en partie sur des comportements d'autoagrégation (SCHELLiNG 1980). Les regroupements affinitaires actifs (départ des classes moyennes vers les couronnes périurbaines) ou passifs (p.ex. le maintien sur place d'une catégorie de population, le vieillissement du parc immobilier) des différents groupes socio-ethniques amplifient des phénomènes ségrégatifs propres au fonctionnement du marché immobilier. La continuité de leurs effets ségrégatifs questionne les modalités de croissance et de développement des régions métropolitaines. Comment conjurer les processus de ségrégation à l'œuvre et les menaces d'éclatement du lien social qui pèsent sur les grandes agglomérations?

\subsection{Ségrégation résidentielle, précarité sociale et xénophobie: les défis de l'intégration}

Les grandes agglomérations sont divisées démographiquement, socialement et culturellement; le régime métropolitain actuel semble accentuer les clivages. Les problèmes de différenciation des espaces urbains n'ont pas, en Suisse, la même acuité que dans d'autres pays européens. Cependant, nous sommes également placés devant le défi de trouver des solutions aux problèmes de précarité des statuts sociaux souvent associés aux processus de ségrégation résidentielle.

Dans l'ensemble de la Suisse, on compte aujourd'hui $500^{\prime} 000$ personnes en situation de précarité. Nous savons qu'en Suisse la question sociale est une question urbaine. C'est dans les agglomérations urbaines et tout particulièrement dans leurs centres et leurs couronnes suburbaines que se concentrent les problèmes de pauvreté et d'exclusion. Traversés par de profonds changements économiques et sociaux, les centres névralgiques des régions métropolitaines sont de plus en plus confrontés à la précarisation du marché de l'emploi (Da Cunha, Leresche \& Vez 1998; Da Cunha \& Both 2004). La désintégration, l'exclusion, l'isolement et la marginalisation sont des phénomènes qui touchent les populations suisses et immigrées. Toutefois, il est avéré que les personnes de nationalité étrangère sont davantage exposées à la précarité, parce qu'elles sont employées dans des secteurs de l'économie plus fragilisés par les fluctuations économiques (p.ex. bâtiment, hôtellerie) et parce que, dans ces secteurs, elles sont plus souvent affectées à des postes peu qualifiés. Globalement, le chômage est trois fois plus élevé chez les étrangers. Les inégalités salariales sont aussi importantes (FLÜCKIGER et al. 2002). L'accès à 
certaines professions tertiaires est pour ainsi dire largement réservé à la population autochtone: dans l'enseignement, la sécurité, les professions judiciaires, les migrants ne représentent que $5 \%$ des postes de travail. La situation des jeunes étrangers en termes de formation et d'accès au marché du travail est particulièrement inquiétante (LISCHER 1997; FANKHAUSERPerez, Käser \& Witzig-Marinho 2003). Comme le soulignent CHAUDET et al. (2003: 373),

«une bonne partie des problèmes sociaux rencontrés par les personnes de nationalité étrangère se rapportent à leur condition et à leur statut, et leur sont donc spécifiques non pas en tant qu'individus, mais en tant que groupe déterminé par son statut».

Marquées par l'afflux de populations aux styles de vie distincts et par la précarité, les sociétés urbaines sont menacées par l'émergence des phénomènes de fragmentation sociale et politique (WIEvIORKA 1997). Le contexte d'affaiblissement généralisé de la «force intégratrice des institutions» (SCHNAPPER 2000) fournit un terrain fertile d'une part aux mouvements de repli communautaire et d'autre part à la diffusion des thèses populistes et xénophobes. Dans l'espace public, le migrant représente le conflit permanent autour du partage d'un bien-être économique fragilisé et d'un patrimoine symbolique confronté à l'altérité. Les étrangers sont devenus un problème récurrent et un enjeu politique national et local, mettant les pouvoirs publics au défi d'un changement majeur de politique migratoire.

\section{Conclusions et perspectives: vers une politique d'agglomération}

Portes d'entrée des migrations internationales, milieux d'installation privilégiés des populations migrantes, mais aussi espaces plus fortement exposés aux incidences sociales des mutations économiques et technologiques, les agglomérations urbaines constituent l'inévitable lieu d'application d'une politique d'intégration sociale et culturelle ouverte sur la nécessité de la construction d'une nouvelle citoyenneté. Les communes des grandes agglomérations ont un rôle majeur à jouer dans la recherche de solutions pratiques aux problèmes d'intégration parfois perçus de manière caricaturale par une partie de la classe politique, des moyens de communication et de l'opinion publique. Le propre d'une politique publique est de gérer en permanence les représentations, les préjugés et les décalages ou les conflits entre différents groupes sociaux ou secteurs de la société (Muller 1990). Bien que leur marge de manœuvre soit limitée, les communes et les villes suisses assument déjà des tâches d'intégration sociale et culturelle. La société tout entière aura tout à gagner du renforcement des conditions-cadre de leur action et des moyens financiers disponibles.

\subsection{Wanted but not welcome: de l'immigré au citoyen}

La représentation que les pouvoirs publics se sont fait du travailleur immigré et de la place qu'il doit occuper dans la société, n'a pas été guidée prioritairement par le souci de l'intégration. La situation qui prévaut jusque dans les années 1990 s'inscrit essentiellement dans une logique économique de contrôle et de gestion des flux de main-d'œuvre. Les migrants sont recherchés comme facteurs de régulation du marché du travail, mais pas forcément bienvenus en tant que minorités ethniques. Dès 1963, le Conseil fédéral recommande de ne pas admettre de travailleurs saisonniers venant du Portugal et de la Turquie et des pays situés hors d'Europe parce que leur «assimilation est difficile, sinon impossible». C'est pour les raisons identiques que le Conseil fédéral limite en 1991 l'admission de personnes originaires de ce qu'il est convenu d'appeler l'ex-Yougoslavie. La politique des trois cercles exprime une vision qui rappelle à ceux-là même qui parviennent à pénétrer la frontière qu'il leur manque quelque chose pour atteindre les qualités attendues du migrant assimilé.

\section{Piguet (2004: 70) souligne que}

«la constellation des facteurs qui caractérise la politique migratoire suisse en ce début du XXI's siècle s'avère fort différente de celle qui prévalait dans les années 1960».

La nouvelle loi sur les étrangers, en chantier depuis 1998, pose les termes d'un compromis bien helvétique entre contrôle et intégration. Pourtant, dans les délibérations parlementaires récentes, une tendance très nette s'est dessinée, qui va à l'encontre des exigences fondamentales formulées par la COMMISSION FÉDÉRALE DES ÉTRANGers (CFE, 1996) à l'égard de la politique d'intégration. Dans une missive adressée aux parlementaires, la CFE plaide notamment pour un renforcement de la politique de l'intégration et pour que l'égalité des droits soit appliquée à toutes les personnes admises dans notre pays.

Les hésitations de la classe politique sont souvent relayées par les médias qui reflètent et parfois alimentent les frustrations d'une partie non négligeable de citoyens suisses, plus souvent d'origine modeste, qui se laisse convaincre par l'argumentaire xénophobe (ChAudet et al. 2003). Les arguments des mouvements populistes qui thématisent le problème des étrangers comme une ressource politique et un levier du pouvoir ne sont pas uniquement rhétoriques: ils explorent les ambiguïtés d'un discours officiel qui peine à reconnaître que la Suisse est devenue un pays d'immigration et une société pluriculturelle. Dans tous les cas, la récurrence et la montée en puissance des mouvements populistes incitent à prendre au sérieux les témoignages de mécontentement et à chercher des solutions capables de prévenir de nouvelles ruptures du lien social. Comme le souligne Haug (1995: 25), 
«les questions qui ont trait à la scolarisation, à la formation ainsi qu'à l'intégration politique et économique des étrangers nés dans notre pays vont acquérir une importance croissante. Maintenant, il est indispensable de préparer consciemment et activement l'intégration sociale, politique et économique des étrangers dans la société suisse.»

A l'ère des migrations, les nations modernes sont placées devant le défi d'intégrer toutes les populations en une communauté des citoyens (SCHNAPPER 2000).

Les questions portant sur l'utilité économique des immigrés, sur leur rôle régulateur du marché du travail ou sur les coûts sociaux entraînés par leur présence cède progressivement la place aux interrogations relatives à la gestion des tensions identitaires dans un climat de précarisation économique et sociale. Dans l'ordre du politique, la question de la cohabitation de personnes ayant des origines multiples et la question sociale ne peuvent plus être dissociées. Les politiques d'intégration doivent assurer l'accès à des ressources culturelles, sociales et politiques permettant de renforcer l'autonomie et la participation de tous à la vie collective. La reconnaissance du pluralisme culturel dans le cadre d'une politique d'intégration doit être étroitement articulée à la mise en œuvre d'une politique de prévention de la précarité en faveur des groupes de population, suisses et immigrés, les plus défavorisés. C'est ce que soulignent aussi dans leur «Charte pour l'intégration», les organisations représentatives des communautés migrantes de Suisse, plaidant ainsi pour une «nouvelle citoyenneté fondée sur l'égale dignité de tous» (FORUM POUR L'INTÉGRATION DES MIGRANTES ET DES MIGRANTS 2005).

\subsection{Préparer activement l'intégration: des actions transversales à l'échelle des agglomérations}

A partir du milieu des années 1980, certains pays européens mettent en œuvre des politiques urbaines pour améliorer le cadre de vie et lutter contre l'exclusion sociale, la pauvreté et les violences urbaines et pour tenter de gérer les différences socio-culturelles liées à l'ethnicisation des sociétés urbaines (DONZELOT \& Estebe 1994; Chaline 1997; Moore 2001). Ces politiques partent de l'idée que la prévention des multiples fractures sociales passe par une action globale liant plus intimement des interventions sectorielles, jusqu'alors trop cloisonnées, au niveau p.ex. de la création d'emplois, de l'éducation et de la formation, de l'aide sociale, du logement, de l'urbanisme. L'ambition affichée de ces actions est de mener une véritable politique de transversalité par la mise en œuvre de ressources et de dispositifs partenariaux et contractuels locaux servant de cadre à l'intervention des professionnels de l'action sociale, du logement et de l'aménagement urbain. Ces interventions n'ont pas toujours produit les résultats escomptés, mais si critiquées et si différentes dans leurs effets soient-elles, elles constituent des expériences novatrices pouvant inspirer l'action en faveur de l'intégration à l'échelle des agglomérations urbaines. Ainsi, il devient de plus en plus tentant de lier l'action en faveur de l'intégration sociale et culturelle à une politique plus globale d'aménagement urbain.

En Suisse, on ne peut pas parler de l'existence d'une politique de la ville. Tout se passe comme si la société refusait de voir dans les villes le territoire de sa propre existence et le lieu majeur d'expression du lien social. La ville en tant que «commune» a toujours signifié la possibilité de trouver quelque chose de commun à toutes les différences et à toutes les multiplicités. Face aux polarisations identitaires, à la persistance de multiples pauvretés, au morcellement des acteurs et des dispositifs de l'action sociale, il convient de réfléchir à l'opportunité d'une action collective globale, concertée et partenariale en faveur de l'intégration des différents groupes de population. AREND (2003) montre comment la ville de Zurich a mis en œuvre des mesures d'urbanisme, de politique du marché du logement et d'aménagement destinées à intégrer des immigrants, à promouvoir les relations interculturelles et à maîtriser la xénophobie. Un rapport récent de la CONFÉRENCE TRIPARTITE SUR LES AGGLOMÉRATIONS (2004: 3) invite notamment les communes à

«renforcer la collaboration transsectorielle horizontale et verticale ainsi que la collaboration avec les acteurs non étatiques concernés, afin d'améliorer les conditions-cadre du processus d'intégration du point de vue institutionnel».

\subsection{Renforcer les conditions-cadre, expliciter la commande publique}

La politique de la ville dont la Suisse semble avoir besoin serait un agencement localisé d'interventions sectorielles élaborées à différentes échelles de décision (communes, cantons, Confédération). Elle serait aussi une nouvelle façon d'aborder la question de l'intégration à partir d'une métamorphose facilitant la nécessaire mise en réseau à l'échelle intercommunale des dispositifs d'intervention sectoriels: p.ex. lutte contre l'échec scolaire, logement social, aide familiale, prévention de la toxicodépendance, insertion sociale et professionnelle, intégration des étrangers. La question de l'intégration est celle du renforcement du lien social. Elle est liée à l'amélioration de l'accès social et spatial et à celle de l'offre d'un environnement construit de qualité. Il y a là tout un champ ouvert à la réflexion sur le développement urbain et à l'articulation de l'action des communes, des cantons et de la Confédération, dans le cadre des politiques d'agglomération orientées vers un triple objectif: l'insertion sociale et professionnelle des groupes les plus défavorisés, la reconnaissance de la diversité culturelle dans l'espace public, l'élargissement de la citoyenneté. 
De manière plutôt ponctuelle que systématique et coordonnée, plusieurs organismes fédéraux ont pour mission de promouvoir l'intégration de la population étrangère. Jusqu'à maintenant, la COMMISSION FÉDÉRALE DES ÉTRANGERS (1996) a pu jouer un rôle majeur de médiateur entre les autorités fédérales d'une part, les services d'aide aux étrangers et les commissions cantonales ou locales oeuvrant en faveur de l'intégration et les associations d'étrangers, d'autre part. Une partie considérable du travail d'intégration s'effectue aussi par les Chambres cantonales, les bureaux communaux et différentes organisations de droit public ou privé. Est-ce que ces interventions souvent dispersées, «bricolées», et avec peu de moyens financiers, peuvent faire évoluer les conditions-cadre actuelles vers une véritable politique publique permettant de faire face aux défis de la précarité dans une Suisse pluriculturelle? La question reste ouverte. Si la nécessité d'approches transversales est de plus en plus visible, la commande publique, dans laquelle pourrait s'inscrire une politique d'intégration à l'échelle des agglomérations, reste implicite. Afin de pouvoir aborder efficacement les défis de la précarité et de l'intégration culturelle, les agglomérations doivent disposer de structures permettant de mettre en réseau les efforts dispersés réalisés par les cantons et les communes et d'assurer parallèlement la coordination avec les offices fédéraux. La politique d'intégration doit impliquer les différents échelons de l'Etat et la société civile dans son ensemble. Les agglomérations sont appelées à mettre en œuvre des structures institutionnelles capables de se doter d'une représentation transparente du processus de différenciation sociale et culturelle des espaces urbains, d'approches prospectives des enjeux sectoriels (p.ex. travail, école, logement) de l'intégration et d'options urbanistiques créatrices de lien social.

\section{Bibliographie}

AREND, M. (2003): La planification urbaine et la politique du marché du logement peuvent-elles contribuer à une meilleure intégration des migrants? - In: WICKER, H.-R., FibBI, R. \& W. HAUG (dir.): Les migrations et la Suisse. - Zurich: Seismo: 227-244.

Ascher, F. (1995): Métapolis ou l'avenir des villes. Paris: Odile Jacob.

Chaline, C. (1997): Les politiques de la ville. - Paris: Presses Universitaires de France.

Chaudet, I., Regamey, C. \& B. Rosende Haver (2003): Les réponses aux problèmes sociaux des personnes de nationalité étrangère. - In: WICKER, H.-R., FIBBI, R. \& W. HAUG (dir.): Les migrations et la Suisse. - Zurich: Seismo: 357-375.

COMMISSION FÉDÉRALE DES ÉTRANGERS (1996): Esquisse pour un concept d'intégration. - Berne: Commission fédérale des étrangers.

CONFÉRENCE TRIPARTITE SUR LES AGGLOMÉRATIONS (2004): Recommandations relatives à la politique des étran- gers et d'intégration. - Berne: Conférence tripartite sur les agglomérations.

Corboz, A. (2000): La Suisse comme hyperville. Paris: Le visiteur 6: 112-129.

Da Cunha, A. \& J.-F. Borth (2004): Métropolisation, villes et agglomérations. Structures socio-démographiques des espaces urbains. - Neuchâtel: Office fédéral de la statistique.

Da Cunha, A., Leresche, J.-P. \& I. Vez (1998) : La pauvreté urbaine. Le lien et les lieux. - Lausanne: Réalités sociales.

Da Cunha, A. et al. (2004): Mobilité résidentielle, aspirations des ménages et transformations de l'habitat: l'agglomération lausannoise. - Lausanne: Rapport de recherche de l'Observatoire de la ville et du développement durable, Institut de géographie de l'Université de Lausanne.

Donzelot, J. \& P. Estebe (1994): L'Etat animateur. Essai sur la politique de la ville. - Paris: Editions Esprit.

FanKhauser-Perez, S., KäSer, B. \& A. Witzig-Marinho (2003): Les familles d'immigrés et le monde du travail. - In:Terra Cognita. Revue suisse de l'intégration et de la migration 3: 98-99.

FLÜCKIGER, Y., NEJADAN, M.Z. et al. (2002): Intégration de la population étrangère en Suisse: aspects économiques et sociaux. - Rapport PNR 39 Migrations et relations interculturelles, Berne: Fonds National Suisse.

FORUM POUR L'INTÉGRATION DES MIGRANTES ET DES MIGRANTS (2005): Charte pour l'intégration du FIMM Suisse. - Berne: Forum pour l'intégration des migrantes et des migrants Suisse.

Haug, W. (1995): La Suisse: Terre d'immigration, société multiculturelle. Eléments pour une politique de migration. - Neuchâtel: Office fédéral de la statistique.

Huissoud, T. et al. (1999): Structures et tendances de la différenciation dans les espaces urbains en Suisse. - Rapport PNR 39 Migrations et relations interculturelles, Berne: Fonds National Suisse et Rapport de recherche 145, Lausanne: Institut de recherche sur l'environnement construit (IREC), Ecole polytechnique fédérale (EPFL).

Lischer, R. (1997): Intégration: une histoire d'échecs. - Neuchâtel: Office fédéral de la statistique.

Martens, A. \& M. Vervaeke (1997): La polarisation sociale des villes européennes. - Paris: Anthropos.

Moore, D. (2001): Ethnicité et politique de la ville en France et en Grande-Bretagne. - Paris: L'Harmattan. Muller, P. (1990): Les politiques publiques. - Paris: Presses Universitaires de France.

Piguet, E. (2004): L'immigration en Suisse. - Lausanne: Presses polytechniques et universitaires romandes.

Schelling, T. (1980): La tyrannie des petites décisions. - Paris: Presses Universitaires de France.

SCHNAPPER, D. (2000): Qu'est-ce que la citoyenneté? Paris: Gallimard. 
WANNer, P. (2004): Migration et intégration. Populations étrangères en Suisse. - Neuchâtel: Office fédéral de la statistique.

Wicker, H.-R., FibBi, R. \& W. HAUg (dir.) (2003): Les migrations et la Suisse. - Zurich: Seismo.

Wieviorka, M. (1997): Une société fragmentée. Le multiculturalisme en débat. - Paris: La Découverte.

Résumé: Métropolisation, migrations internationales et pluralité des espaces sociaux: les agglomérations suisses face au défi de l'intégration

La Suisse est traversée par des tensions engendrées par les réalités d'une société urbaine pluriculturelle faite de diversités, mais aussi de clivages et d'inégalités. Le fait migratoire et l'intégration des populations étrangères constituent des enjeux démographiques, économiques et sociaux, mais aussi des objets récurrents de passions et de débats politiques. Portes d'entrée des migrations internationales, les grandes agglomérations suisses sont placées devant le défi de trouver des programmes d'action visant l'intégration de populations aux origines, aux cultures et aux religions de plus en plus diversifiées. Le présent article décrit ce type de situations tel qu'il se présente actuellement en Suisse et s'interroge sur les moyens de contrer les processus de ségrégation à l'œuvre. Il précise que la question sociale et celle du respect des droits et coutumes de personnes d'origine diverse ne peuvent plus être dissociées. La législation publique doit intégrer une telle perspective. Les agglomérations urbaines sont appelées à mettre en œuvre des actions transversales visant à gérer la diversité, à renforcer le lien social et à élargir l'exercice de la citoyenneté.

\section{Abstract: Metropolization, international migration and social spaces: Swiss urban areas and the challenge of integration}

Across Switzerland, tensions are apparent, provoked not only by the realities of an urban and diverse multicultural society, but also by policies that lead to inequality and social differentiation. Migration and the integration of foreign populations are not only real demographic, economic and social issues, they also serve as popular and emotionally laden topics in political debates. The large urban areas of Switzerland are the entry point for international migrants. These urban areas face the challenge of finding the means of integrating newcomers from diverse cultures and religions. This article describes the situation as it is found in Switzerland and discusses ways of preventing segregation of newcomers. It supports the idea that social integration and the respect of the rights and habits of people of diverse origins who must live together are two questions which can no longer be dissociated. Public policy must be developed from this perspective. Urban areas are encouraged to implement cross-cutting action to manage diversity, reinforce social links and enhance the practice of citizenship.

\section{Zusammenfassung: Metropolisation, internationale Migration und Pluralität sozialer Räume: die Schweizer Agglomerationen angesichts der Herausforderung der Integration}

Wie in allen europäischen Nationen gibt es in der Schweiz Spannungen, die von einer urbanen multikulturellen Gesellschaft mit ihren Verschiedenheiten aber auch von ihren Abgrenzungen und Ungleichheiten herrühren. Die Migration und die Integration fremder Volksgruppen sind demographische, wirtschaftliche und soziale Herausforderungen, aber sie sind auch immer wiederkehrende Themen von Emotionen und politischen Auseinandersetzungen. Als Eingangstore der internationalen Migration werden die grossen schweizerischen Agglomerationen vor das Problem gestellt, Aktionsprogramme zu kreieren, um die Bevölkerungsgruppen verschiedener Herkunft, Kulturen und Religionen zu integrieren. Dieser Artikel beschreibt die Situation wie sie in der Schweiz auftritt und behandelt die Möglichkeiten, Segregationsprozesse betreffend dieser Neuankömmliche zu vermeiden. Der Beitrag unterstützt die Idee, dass die soziale Integration und der Respekt vor den Rechten und Gepflogenheiten von Menschen unterschiedlicher Herkunft, die miteinander leben müssen, nicht länger voneinander getrennt betrachtet werden können. Die Rechtsordnung muss aus dieser Perspektive heraus entwickelt werden. Städtische Gebiete sind aufgerufen, verbindende Massnahmen zu ergreifen, um mit der Verschiedenheit umzugehen, soziale Bindungen zu stärken und die Praxis der Staatsbürgerschaft auszuweiten.

Prof. Dr. Antonio Da Cunha, Observatoire universitaire de la ville et du développement durable, Institut de géographie, Université de Lausanne, Quartier Dorigny, Bâtiment Humense, $\mathrm{CH}-1015$ Lausanne. e-mail: Antonio.DaCunha@unil.ch

\section{Manuskripteingang/received/manuscrit entré le} 12.5.2005

Annahme zum Druck/accepted for publication/accepté pour l'impression: 5.9.2005 